\title{
A topological version of the Ambrosetti-Prodi theorem
}

\author{
by Bogdan PrzeradzKi (Łódź)
}

\begin{abstract}
The existence of at least two solutions for nonlinear equations close to semilinear equations at resonance is obtained by the degree theory methods. The same equations have no solutions if one slightly changes the right-hand side. The abstract result is applied to boundary value problems with specific nonlinearities.
\end{abstract}

1. Introduction. The question of the solvability of nonlinear equations has been extensively studied since the famous work of Schauder and Leray. Most of the results give sufficient conditions for the existence of at least one solution to such an equation. However, some equations have the "uninteresting" trivial solution and one should look for nontrivial ones. These can be obtained if we prove the existence of at least two solutions. On the other hand, it is sometimes important to know that an equation has more than one solution somehow localized, positive or negative for instance. After the well-known paper by Ambrosetti and Prodi [2] a lot of results appeared concerning similar equations and using similar ideas. Most authors study particular boundary value problems such as:

$$
\Delta u+f(u)=h(x), \quad u \mid \partial \Omega=0
$$

(comp. [1], [11], [12]);

$$
x^{\prime \prime}+x=f(t, x)+h(t), \quad x(0)=x(\pi)=0
$$

(comp. [4], [5], [3]);

$$
x^{\prime \prime}+c x^{\prime}=f(t, x)+h(t)+s, \quad x(0)=x(T), \quad x^{\prime}(0)=x^{\prime}(T)
$$

(comp. [11], [3]). Assumptions on the nonlinear term make $f$ similar to the model function $f_{0}(x)=x^{2}$. Assertions are usually of the form: there are at least two solutions for some $h$, no solution for other ones and one solution for $h$ belonging to the set separating the above two domains. It is a common feature that equations have two linear approximants for $x \rightarrow+\infty$

1991 Mathematics Subject Classification: 47H15, 34K10.

Key words and phrases: multiple solution, resonance, functional-differential equation. 
and $x \rightarrow-\infty$ and that there exists a linear noninvertible operator "between" them. We use this observation to formulate our problem and result; however, its application to the above typical boundary value problems is impossible. We can (and do) apply the main theorem to boundary value problems for functional-differential equations of a special form.

The approach is based on a method from the series of papers by the present author [7]-[10] where he deals only with the existence of at least one solution. The method seems to be applicable also for nonlinearities jumping over several eigenvalues when we expect more than two solutions (see [4]).

2. An abstract result. Let $X, Y$ and $Z$ be real Banach spaces, $v_{1} \in X^{*}$, the space of bounded linear functionals on $X$; let $N: X \rightarrow Z$ be a nonlinear continuous operator mapping bounded sets into bounded ones, such that there exist two linear bounded operators $L_{+}, L_{-}: X \rightarrow Z$ with the property

$$
\lim _{v_{1}(x) \rightarrow \pm \infty} v_{1}(x)^{-1}\left\|N(x)-L_{ \pm}(x)\right\|=0 .
$$

Let $J: Y \rightarrow Z$ be an injective completely continuous linear operator and let $L_{0}: Y \rightarrow Z$ be a linear bounded operator with $\operatorname{codim}_{Z}\left(\operatorname{im} L_{0}\right)=1$. Take $u_{1} \in Z^{*}$ such that $\operatorname{ker} u_{1}=\operatorname{im} L_{0}$ and fix $h_{0} \in \operatorname{im} L_{0}, h_{1} \in Z$ such that $u_{1}\left(h_{1}\right)=1$. We look for solutions to the equation

$$
L_{0} y=N(J y)+h_{0}+s h_{1},
$$

where $s$ is a real parameter. We work under the following assumptions:

(a) $L_{0}-L_{ \pm} J$ are linear homeomorphisms;

(b) for any $M>0, u_{1}$ is bounded on the sets $N\left(\left\{x:\left|v_{1}(x)\right| \leq M\right\}\right)$, $L_{ \pm}\left\{x:\left|v_{1}(x)\right| \leq M\right\}$

(c)

$$
\liminf _{v_{1}(x) \rightarrow+\infty} v_{1}(x)^{-1} u_{1}\left(L_{+} x\right)>0, \quad \limsup _{v_{1}(x) \rightarrow-\infty} v_{1}(x)^{-1} u_{1}\left(L_{-} x\right)<0 .
$$

Usually, $L_{0}$ is a Fredholm operator of index 0 , but this is not necessary for our purposes.

Theorem. There exists $S_{0}>0$ such that equation (2.2) has no solution for $s \geq S_{0}$ and has at least two solutions for $s \leq-S_{0}$.

Proof. Suppose that there exist sequences $s_{n} \rightarrow+\infty$ and $\left(y_{n}\right) \subset Y$ such that

$$
L_{0} y_{n}=N\left(J y_{n}\right)+h_{0}+s_{n} h_{1} .
$$

Hence $u_{1}\left(N\left(J y_{n}\right)\right)=-s_{n}$, which implies the unboundedness of the sequence $\left(v_{1}\left(J y_{n}\right)\right)$. On the other hand, if there is a subsequence with the property 
$v_{1}\left(J y_{n}\right) \rightarrow \pm \infty$, then

$$
v_{1}\left(J y_{n}\right)^{-1} u_{1}\left(N\left(J y_{n}\right)-L_{ \pm} J y_{n}\right)=-v_{1}\left(J y_{n}\right)^{-1} s_{n}-v_{1}\left(J y_{n}\right)^{-1} u_{1}\left(L_{ \pm} J y_{n}\right)
$$

tends to 0 by (2.1), which is impossible due to assumption (c).

The proof of the second part needs the Leray-Schauder theory. Let us consider a completely continuous map $H_{+}:[0,1] \times X \rightarrow X$ given by

$$
H_{+}(\lambda, x)=\lambda J\left(L_{0}-L_{+} J\right)^{-1}\left(N(x)-L_{+} x\right)+J\left(L_{0}-L_{+} J\right)^{-1}\left(h_{0}+s h_{1}\right)
$$

and a positive number $M$ such that $v_{1}(x) \geq M$ implies

$$
v_{1}(x)^{-1}\left\|J\left(L_{0}-L_{+} J\right)^{-1}\left(N(x)-L_{+} x\right)\right\|<\left(2\left\|v_{1}\right\|\right)^{-1} .
$$

It follows that, for any $\lambda \in[0,1]$, the fixed points $x$ of $H_{+}(\lambda, \cdot)$ satisfy $v_{1}(x)<M$ or

$$
\|x\|<2\left\|J\left(L_{0}-L_{+} J\right)^{-1}\left(h_{0}+s h_{1}\right)\right\| .
$$

On the other hand, if $H_{+}(\lambda, x)=x$, then $x=J y$, where

$$
L_{0} y=\lambda N(J y)+(1-\lambda) L_{+} J y+h_{0}+s h_{1}
$$

and the condition $v_{1}(x) \in[0, M)$ implies

$$
0=\lambda u_{1}(N(x))+(1-\lambda) u_{1}\left(L_{+} x\right)+s .
$$

If we take $S_{0}>\sup \left\{\left|u_{1}(N(x))\right|:\left|v_{1}(x)\right| \leq M\right\}+\sup \left\{\left|u_{1}\left(L_{+} x\right)\right|:\left|v_{1}(x)\right|\right.$ $\leq M\}$ (which is finite due to (b)), then, for $s \leq-S_{0}$, the homotopy $H_{+}$has no fixed points on the boundary of the domain

$$
V_{+}=\left\{x: v_{1}(x)>0,\|x\|<2\left\|J\left(L_{0}-L_{+} J\right)^{-1}\left(h_{0}+s h_{1}\right)\right\|\right\} .
$$

Hence the Leray-Schauder degree $\operatorname{deg}_{\mathrm{LS}}\left(I-H_{+}(\lambda, \cdot), V_{+}, 0\right)$ does not depend on $\lambda \in[0,1]$. But

$$
\operatorname{deg}_{\mathrm{LS}}\left(I-H_{+}(0, \cdot), V_{+}, 0\right)=\operatorname{deg}_{\mathrm{LS}}\left(I, V_{+}, J\left(L_{0}-L_{+} J\right)^{-1}\left(h_{0}+s h_{1}\right)\right)=1,
$$

which means that there exists $x=H_{+}(1, x) \in V_{+}$. Obviously, $x=J y$, where $y$ is a solution to equation (2.2). We can find the second solution by taking the homotopy

$$
H_{-}(\lambda, x)=\lambda J\left(L_{0}-L_{-} J\right)^{-1}\left(N(x)-L_{-} x\right)+J\left(L_{0}-L_{-} J\right)^{-1}\left(h_{0}+s h_{1}\right)
$$

on the set

$$
V_{-}=\left\{x: v_{1}(x)<0,\|x\|<2\left\|J\left(L_{0}-L_{-} J\right)^{-1}\left(h_{0}+s h_{1}\right)\right\|\right\} .
$$

Remarks. $v_{1}: X \rightarrow \mathbb{R}$ need not be a linear functional. In fact, it should satisfy $\left|v_{1}(x)\right| \leq c\|x\|, x \in X$, for a positive $c$, take values tending to $+\infty$ and $-\infty$ and the sets $\left\{x: v_{1}(x)>0\right\},\left\{x: v_{1}(x)<0\right\}$ should be open. Moreover, $v_{1}$ can be defined only on a set admissible for a fixed point index theory (ANR, for instance). In this case, we just replace the Leray-Schauder degree by the appropriate fixed point index. 
3. Applications. Although our theorem has the same roots as the results of Ambrosetti-Prodi and their successors [2], [11], they are incomparable in applications. The nonlinear part $N$ is, in typical boundary value problems, a Nemytskiŭ superposition operator $x \mapsto f(\cdot, x(\cdot))$. However, asymptotic condition (2.1) cannot be expressed by means of properties of the function $f$, except for trivial examples. If one wants (2.1) to hold, then $f$ should depend on $v_{1}(x)$. We present the simplest case in detail.

Let us consider the periodic boundary value problem

$$
x^{\prime}=f\left(t, x, \int_{0}^{T} x(r) d r\right)+h_{0}(t)+s, \quad x(0)=x(T),
$$

where $f:[0, T] \times \mathbb{R}^{2} \rightarrow \mathbb{R}$ is a Carathéodory function in the following sense: $f(t, \cdot, \cdot)$ is continuous for a.e. $t \in[0, T], f(\cdot, x, y)$ is measurable for all $x, y$, and, for any $M>0$, there exist an integrable function $\vartheta_{M} \in L^{1}(0, T)$ and a positive constant $\alpha_{M}$ such that

$$
|f(t, x, y)| \leq \alpha_{M}|x|+\vartheta_{M}(t)
$$

for any $t \in[0, T], x \in \mathbb{R}$ and $|y| \leq M$. In (3.1), $s$ is a real parameter and $h_{0} \in L^{1}(0, T)$ satisfies

$$
\int_{0}^{T} h_{0}(t) d t=0 .
$$

Suppose that $f$ has the following asymptotic property: there exist $\lambda_{+}>0$ and $\lambda_{-}<0$ such that

$$
\lim _{v_{1}(x) \rightarrow \pm \infty} v_{1}(x)^{-1}\left|\int_{0}^{T}\left(f\left(t, x(t), v_{1}(x)\right)-\lambda_{ \pm} x(t)\right) d t\right|=0,
$$

and, for any $M>0$, there is $\beta_{M}>0$ such that

$$
\left|\int_{0}^{T} f\left(t, x(t), v_{1}(x)\right) d t\right| \leq \beta_{M}
$$

for $\left|v_{1}(x)\right| \leq M$. We shall show that our theorem can be applied, which means that (3.1) has no solution for large positive $s$ and has at least two solutions for large negative $s$.

Put $X=Z=L^{1}(0, T), Y=\left\{y \in W^{1,1}(0, T): y(0)=y(T)\right\}$, where $W^{1,1}(0, T)$ stands for the Sobolev space of absolutely continuous functions on $[0, T]$ with the norm

$$
\|y\|=\int_{0}^{T}\left(|y(t)|+\left|y^{\prime}(t)\right|\right) d t .
$$


Set

$$
\begin{gathered}
L_{0} y=y^{\prime}, \quad J y=y, \quad v_{1}(x)=u_{1}(x)=\int_{0}^{T} x(t) d t, \\
N(x)(t)=f\left(t, x(t), v_{1}(x)\right), \quad\left(L_{ \pm} x\right)(t)=\lambda_{ \pm} x(t) .
\end{gathered}
$$

One can easily verify that $u_{1}$ is chosen well, $J$ is completely continuous by the Sobolev Embedding Theorem, $N: L^{1}(0, T) \rightarrow L^{1}(0, T)$ is continuous and maps bounded sets into bounded ones by (3.2), condition (2.1) is a consequence of (3.3). In order to check assumption (a), let us notice that the equations $x^{\prime}=\lambda_{ \pm} x$ have no $T$-periodic solutions. Condition (3.4) implies (b). Finally, the fractions under the limits in (c) equal $\lambda_{+}>0$ and $\lambda_{-}<0$, respectively.

One can replace unpleasant conditions (3.3), (3.4) by simpler ones (without integrals) but then important examples will not be covered. For instance,

$$
f(t, x, y)=\chi(y) x+g(t, x),
$$

where $\chi: \mathbb{R} \rightarrow \mathbb{R}$ is a continuous function with

$$
\lambda_{ \pm}=\lim _{y \rightarrow \pm \infty} \chi(y)
$$

and $g$ is a Carathéodory function bounded by an integrable one, satisfies our assumptions.

We can study the same boundary value problem (3.1) using completely different assumptions. Let $f:[0, T] \times \mathbb{R}^{2} \rightarrow \mathbb{R}$ be a continuous function and $f(0, x, y)=f(T, x, y)$ for all $x, y \in \mathbb{R}$; let $h_{0}$ be a continuous real function with the vanishing integral over $[0, T]$. Condition (3.3) is replaced by

$$
\lim _{y \rightarrow \pm \infty} \frac{f(t, x, y)}{y}=\lambda_{ \pm}(t),
$$

uniformly with respect to $t, x$. We do not change condition (3.4). The continuous functions $\lambda_{ \pm}:[0, T] \rightarrow \mathbb{R}$ are supposed to have positive and negative integrals over $[0, T]$, respectively.

Let $X=Z=C[0, T], Y=\left\{y \in C^{1}[0, T]: y(0)=y(T)\right\}$, and let $L_{0}, J$, $u_{1}, v_{1}$ and $N$ be as above. We can put $\left(L_{ \pm} x\right)(t)=\lambda_{ \pm}(t) v_{1}(x)$. Asymptotic property (2.1) is a direct consequence of (3.6), the proof of (a) is given below, (b) follows from (3.4) and the definition of $L_{ \pm}$. In (c), we have under the limits

$$
\int_{0}^{T} \lambda_{ \pm}(t) d t
$$

that is positive and negative, respectively. 
Pr o of of (a). We shall find the only solution to the boundary value problem

$$
x^{\prime}=\int_{0}^{T} x(s) d s \cdot \lambda(t)+z(t), \quad x(0)=x(T),
$$

where $\lambda$ is a fixed function with a nonzero integral over $[0, T]$, and $\lambda, z \in$ $C[0, T]$. Suppose that $x$ is a solution and $c=\int_{0}^{T} x(t) d t$. Then

$$
x(t)=x(0)+c \int_{0}^{t} \lambda(s) d s+\int_{0}^{t} z(s) d s
$$

and the periodic condition gives

$$
c=\left(\int_{0}^{T} \lambda(s) d s\right)^{-1} \int_{0}^{T} z(s) d s .
$$

If we integrate (3.8) over $[0, T]$ and apply the Fubini Theorem, we shall get

$$
c=T x(0)+c \int_{0}^{T}(T-s) \lambda(s) d s+\int_{0}^{T}(T-s) z(s) d s .
$$

Applying (3.9), we obtain

$$
\left(1+\int_{0}^{T} s \lambda(s) d s\right) c=T x(0)-\int_{0}^{T} s z(s) d s .
$$

Hence, we can express $x(0)$ and $c$ by means of

$$
\begin{aligned}
z_{0}=\int_{0}^{T} z(s) d s, & z_{1}=\int_{0}^{T} s z(s) d s, \\
\lambda_{0}=\int_{0}^{T} \lambda(s) d s, & \lambda_{1}=\int_{0}^{T} s \lambda(s) d s .
\end{aligned}
$$

That is,

$$
x(0)=T^{-1}\left(c\left(1+\lambda_{1}\right)+z_{1}\right), \quad c=\lambda_{0}^{-1} z_{0},
$$

and we substitute it in (3.8). It follows that a solution of (3.7) is unique and continuously expressed by $z_{0}$.

The following function satisfies our assumptions:

$$
f(t, x, y)=\chi(t, y)+g(t, x),
$$

where $\chi, g:[0, T] \times \mathbb{R} \rightarrow \mathbb{R}$ are continuous, $\chi(0, y)=\chi(T, y), g(0, x)=$ $g(T, x), g$ is bounded and

$$
\lim _{y \rightarrow \pm \infty} y^{-1} \chi(t, y)=\lambda_{ \pm}(t)
$$

uniformly in $t$. 
In the same way one can study the periodic problem for the second order differential equation

$$
x^{\prime \prime}=f\left(t, x, \int_{0}^{T} x(r) d r\right)+h_{0}(t)+s, \quad x(0)=x(T), \quad x^{\prime}(0)=x^{\prime}(T) ;
$$

the Dirichlet problem

$x^{\prime \prime}+m^{2} x=f\left(t, x, \int_{0}^{\pi} x(r) \sin m r d r\right)+h_{0}(t)+s \sin m t, \quad x(0)=x(\pi)=0 ;$

or the Neumann problem

$$
x^{\prime \prime}=f\left(t, x, \int_{0}^{T} x(r) d r\right)+h_{0}(t)+s, \quad x^{\prime}(0)=x^{\prime}(T)=0 .
$$

Particular attention should be paid to (a) which needs new bounds on $\lambda_{ \pm}$ in both cases.

4. Functional-differential equations. We study the general functional-differential equation

$$
\left.x^{\prime}=f\left(t, x_{t}, v_{1}(x)\right)\right)+h_{0}(t)+s h_{1}(t),
$$

with the boundary condition

$$
A x_{0}+B x_{1}=0 .
$$

Here $f:[0,1] \times \mathcal{C} \times \mathbb{R} \rightarrow \mathbb{R}$ is a Carathéodory function, where $\mathcal{C}=C[-r, 0]$, i.e. $f(t, \cdot, \cdot)$ is continuous for a.e. $t, f(\cdot, \phi, y)$ is measurable for all $\phi \in \mathcal{C}$, $y \in \mathbb{R}$, and, for any $M>0$, there exist $\alpha_{M}>0$ and $\vartheta_{M} \in L^{1}(0,1)$ such that

$$
|f(t, \phi, y)| \leq \alpha_{M}\|\phi\|_{\mathcal{C}}+\vartheta_{M}(t)
$$

for $t \in[0,1], \phi \in \mathcal{C},|y| \leq M$ (comp. [6], pp. 10-13). Moreover, $x_{t}(s)=$ $x(t+s)$ for $t \in[0,1], s \in[-r, 0]$, and $x:[-r, 1] \rightarrow \mathbb{R}, v_{1}$ is a linear bounded functional on $C[-r, 1]$ of the form

$$
v_{1}(x)=\int_{-r}^{1} \widetilde{v}_{1}(t) x(t) d t
$$

where $\widetilde{v}_{1} \in L^{\infty}(-r, 1), A$ and $B$ are linear bounded operators in $\mathcal{C}$. Suppose that the boundary value problem

$$
x^{\prime}=z(t), \quad A x_{0}+B x_{1}=0,
$$

for $z \in L^{1}(0,1)$ has a solution $x:[-r, 1] \rightarrow \mathbb{R}$ absolutely continuous on $[0,1]$ if and only if $u_{1}(z)=0$, where $u_{1}$ is a linear bounded functional on $L^{1}(0,1)$, 
i.e.

$$
u_{1}(z)=\int_{0}^{1} \widetilde{u}_{1}(t) z(t) d t, \quad \widetilde{u}_{1} \in L^{\infty}(0,1) .
$$

By applying the considerations from [6], p. 11, it is easy to formulate this property by means of $A$ and $B$. Namely, the range $\operatorname{im} B T$ is closed in $\mathcal{C}$ and

$$
\operatorname{codim}_{\mathrm{im} B T}(\operatorname{im}(A+B S) \cap \operatorname{im} B T)=1
$$

where $S: \mathcal{C} \rightarrow \mathcal{C}$ and $T: L^{1}(0,1) \rightarrow \mathcal{C}$ are linear bounded operators given by

$$
\begin{gathered}
S \phi(s)= \begin{cases}\phi(s+1) & \text { for } s \in[-r,-1], \\
\phi(0) & \text { for } s \in(-1,0],\end{cases} \\
T z(s)= \begin{cases}0 & \text { for } s \in[-r,-1], \\
\int_{0}^{s+1} z(t) d t & \text { for } s \in(-1,0] .\end{cases}
\end{gathered}
$$

The functions $h_{0}, h_{1} \in L^{1}(0,1)$ in $(4.1)$ are chosen in the way that $u_{1}\left(h_{0}\right)=$ $0, u_{1}\left(h_{1}\right)>0$, and $s$ is a real parameter.

The linear asymptotes in (2.1) will be of a special form. Let there exist two functions $\lambda_{ \pm} \in L^{\infty}(0,1)$ such that

$$
\lim _{v_{1}(x) \rightarrow \pm \infty} v_{1}(x)^{-1} \int_{0}^{1}\left|f\left(t, x_{t}, v_{1}(x)\right)-\lambda_{ \pm}(t) x(t-r)\right| d t=0 .
$$

The boundary value problem (4.1)-(4.2) is suitable for our framework if we take $X=C[-r, 1], Z=L^{1}(0,1), Y=\left\{y \in X:\left.y\right|_{[0,1]}\right.$ is absolutely continuous, $\left.A y_{0}+B y_{1}=0\right\}, L_{0} y=y^{\prime}, N(x)(t)=f\left(t, x_{t}, v_{1}(x)\right), J y=y$, $\left(L_{ \pm} x\right)(t)=\lambda_{ \pm}(t) x(t-r)$. Then condition (4.4) is a reformulation of (2.1). Assumption (a) can be satisfied if the operators $A+B S_{ \pm}: \mathcal{C} \rightarrow \mathcal{C}$ are invertible, where

$$
S_{ \pm} \phi(s)= \begin{cases}\phi(s+1) & \text { for } s \in[-r,-1], \\ \phi(0)+\int_{0}^{s+1} \lambda_{ \pm}(t) \phi(t-1) & \text { for } s \in(-1,0] .\end{cases}
$$

In order to get (b), we assume that, for any $M>0$,

$$
\sup _{\left|v_{1}(x)\right| \leq M}\left|\int_{0}^{1} \widetilde{u}_{1}(t) f\left(t, x_{t}, v_{1}(x)\right) d t\right|<\infty,
$$

and that

$$
\widetilde{v}_{1}(t)= \begin{cases}\widetilde{u}_{1}(t+r) \lambda_{ \pm}(t+r) l_{ \pm} & \text {for } t \in[-r,-r+1], \\ 0 & \text { for } t>-r+1,\end{cases}
$$

where $l_{ \pm}$are some constants. If $l_{+}>0$ and $l_{-}<0$, then condition (c) will be satisfied as well. Under the above assumptions, the boundary value problem 
(4.1)-(4.2) has no solution for large $s>0$ and has at least two solutions for large $s<0$.

If we take $r=1, A=-B=I$ (the identity operator), then $\widetilde{u}_{1}(t)=1$, so we should have

$$
\widetilde{v}_{1}(t)= \begin{cases}\lambda_{ \pm}(t+1) \cdot l_{ \pm} & \text {for } t \in[-1,0] \\ 0 & \text { for } t \in(0,1]\end{cases}
$$

This means that

$$
\lambda_{+}(t)=l_{-}^{-1} l_{+} \lambda_{-}(t)
$$

for $t \in[0,1]$.

Let $f(t, \phi, y)=\chi(t, y) \phi(-1)+g(t, \phi)$, where $\chi:[0,1] \times \mathbb{R} \rightarrow \mathbb{R}$ is a continuous function such that

$$
\lim _{y \rightarrow \pm \infty} \chi(t, y)=\lambda_{ \pm}(t)
$$

uniformly with respect to $t$, and $g:[0,1] \times C[-1,0] \rightarrow \mathbb{R}$ is a Carathéodory function such that $|g(t, \phi)| \leq \vartheta(t)$ for any $t$ and $\phi$, provided that $\vartheta \in L^{1}(0,1)$. Then all the assumptions are satisfied if the functions $\lambda_{ \pm}$satisfy (4.5) with $l_{+}$positive and $l_{-}$negative.

\section{References}

[1] H. Amann and P. Hess, A multiplicity result for a class of elliptic boundary value problems, Proc. Roy. Soc. Edinburgh 84A (1979), 145-151.

[2] A. Ambrosetti and G. Prodi, On the inversion of some differentiable mappings with singularities between Banach spaces, Ann. Mat. Pura Appl. 93 (1973), 231-247.

[3] C. Fabry, J. Mawhin and M. Nkashama, A multiplicity result for periodic solutions of forced nonlinear second order differential equations, Bull. London Math. Soc. 18 (1986), 173-180.

[4] A. C. Lazer and P. J. McKenna, On the number of solutions of a nonlinear Dirichlet problem, J. Math. Anal. Appl. 84 (1981), 282-284.

[5] - - - On a conjecture related to the number of solutions of a nonlinear Dirichlet problem, Proc. Roy. Soc. Edinburgh 95A (1983), 275-283.

[6] J. Mawhin, Topological Degree Methods in Nonlinear Boundary Value Problems, CBMS Regional Conf. Ser. in Math. 40, Amer. Math. Soc., Providence, R.I., 1977.

[7] B. Przeradzki, An abstract version of the resonance theorem, Ann. Polon Math. 53 (1991), 35-43.

[8] - A new continuation method for the study of nonlinear equations at resonance, J. Math. Anal. Appl. 180 (1993), 553-565.

[9] —, Nonlinear boundary value problems at resonance for differential equations in Banach spaces, Math. Slovaca, to appear.

[10] - Three methods for the study of semilinear equations at resonance, Colloq. Math. 66 (1993), 109-129. 
[11] B. Ruf, Multiplicity results for nonlinear elliptic equations, in: Proc. of the Spring School, Litomyšl, 1986, Teubner-Texte zur Math. 93, 1986, 109-138.

[12] S. Solimini, Multiplicity results for a nonlinear Dirichlet problem, Proc. Roy. Soc. Edinburgh 96A (1984), 331-336.

Institute of Mathematics

University of Łódź

Banacha 22

90-238 Łódź, Poland

E-mail: przeradz@krysia.uni.lodz.pl

Reçu par la Rédaction le 20.3.1995 